\title{
The vertebrate brain, evidence of its modular organization and operating system: insights into the brain's basic units of structure, function, and operation and how they influence neuronal signaling and behavior
}

\author{
Morris H. Baslow* \\ Center for Neurochemistry, Nathan S. Kline Institute for Psychiatric Research, Orangeburg, NY, USA
}

\section{Edited by:}

Riccardo Brambilla, San Raffaele Scientific Institute and University, Italy

Reviewed by:

Osborne F. Almeida, University of Minho, Portugal

Akshay Anand, Post Graduate Institute of Medical Education and Research,

India

\section{*Correspondence:}

Morris H. Baslow, Center for

Neurochemistry, Nathan S. Kline

Institute for Psychiatric Research, 140

Old Orangeburg Road, Orangeburg, NY

10962, USA.

e-mail: baslow@nki.rfmh.org
The human brain is a complex organ made up of neurons and several other cell types, and whose role is processing information for use in eliciting behaviors. However, the composition of its repeating cellular units for both structure and function are unresolved. Based on recent descriptions of the brain's physiological "operating system,"a function of the tri-cellular metabolism of $\mathrm{N}$-acetylaspartate (NAA) and N-acetylaspartylglutamate (NAAG) for supply of energy, and on the nature of "neuronal words and languages" for intercellular communication, insights into the brain's modular structural and functional units have been gained. In this article, it is proposed that the basic structural unit in brain is defined by its physiological operating system, and that it consists of a single neuron, and one or more astrocytes, oligodendrocytes, and vascular system endothelial cells. It is also proposed that the basic functional unit in the brain is defined by how neurons communicate, and consists of two neurons and their interconnecting dendritic-synaptic-dendritic field. Since a functional unit is composed of two neurons, it requires two structural units to form a functional unit. Thus, the brain can be envisioned as being made up of the three-dimensional stacking and intertwining of myriad structural units which results not only in its gross structure, but also in producing a uniform distribution of binary functional units. Since the physiological NAA-NAAG operating system for supply of energy is repeated in every structural unit, it is positioned to control global brain function.

Keywords: behavior, brain, cognition, language, learning, memory, neurons, signaling

\section{INTRODUCTION}

The vertebrate brain is a complex organ made up of neurons and a few other cell types, and whose role is the processing of information for use in elicitation of behaviors. However, the nature of neural coding and the principle of its operation are incompletely known (Djurfeldt et al., 2008). In addition, the precise nature of its hierarchical modular repeating cellular units for both structure and function are also unresolved (Meunier et al., 2009). In this article, evidence of the metabolic and cellular organization of the brain's physiological operating system, and how this system interacts with neuronal codes is presented, and it is proposed that this operating system and its interaction with neuronal codes defines the brain's basic units of structure and function.

\section{NEURONAL ENERGY REQUIREMENTS AND THE TRI-CELLULAR METABOLISM OF $N$-ACETYLASPARTATE AND N-ACETYLASPARTYLGLUTAMATE}

The brain's information processing ability relies on its receiving, and then transmitting, meaningful frequency-encoded electrophysiological signals that originate in a variety of sensing

Abbreviations: Ac, acetate; AcCoA, acetyl-coenzyme A; AQP4, aquaporin 4; Asp, aspartate; CD, Canavan disease; DSD, dendritic-synaptic-dendritic; ECF, extracellular fluid; Gj, gap junction; Glc, glucose; Gln, glutamine; Glu, glutamate; GRM3, metabotropic glutamate receptor-3; MWP, molecular water pump; NAA, $N$-acetylaspartate; NAAG, $N$-acetylaspartylglutamate; $\mathrm{P}$, pause; $\mathrm{S}$, spike; $S y n$, synapse; $T$, tight junction. neurons, both internally and externally, and without which input normal cognitive processing cannot occur (Baslow and Guilfoyle, 2006). In order to carry out their information processing functions, neurons require large amounts of energy, and their lifeline for this energy is the circulatory system upon which they rely for a continuous supply of nutrients and for removal of metabolic waste products and heat. Thus, the well being of both the brain and the whole organism depend on continuous and rapid interactions between neurons and the vascular system. For this purpose, neurons synthesize a neurotransmitter, $\mathrm{N}$-acetylaspartylglutamate (NAAG), and release it to extracellular fluid (ECF) upon stimulation. NAAG is targeted to astrocytes where it is hydrolyzed releasing glutamate (Glu) that then stimulates the astrocytes to release second messengers to vascular endothelial cells which in turn initiate focal hyperemic responses (Baslow and Guilfoyle, 2006). The hydrolysis of NAAG also liberates $\mathrm{N}$-acetylaspartate (NAA) that cannot be further metabolized by astrocytes, and which is transported via ECF to oligodendrocytes for its hydrolysis. It has been proposed that the stimulation-induced release of NAAG serves as a homeostatic neuron to vascular system mechanism that maintains the ability of neurons to obtain energy as needed and thus to continuously receive and transmit meaningful frequency-encoded messages (Baslow and Guilfoyle, 2007). 


\section{NEURONAL CODES}

The nature of neuronal codes has been considered, and a twocell biosemiotic model of inter-neuronal communication has been developed (Baslow, 2009). In that model, it was reasoned that although a single neuron can generate meaningful "words" in a specific language format, that the information would be of no practical use unless transmitted and its meaning understood by a second neuron. Therefore, it was proposed that two neurons are required for communication, and further, that their shared dendritic-synaptic-dendritic (DSD) field contained all of the structural and biochemical mechanisms that allow for learning and memory formation. It was also proposed that these binary inter-neuronal transmissions were the basis for all levels of internal brain and inter-organism communications.

\section{THE NATURE OF THE HIERARCHICAL MODULAR UNITS FOR BOTH STRUCTURE AND FUNCTION}

The two-cell biosemiotic model, when coupled with identification of the tri-cellular metabolism of NAA and NAAG, and the homeostatic physiological role of NAAG in regulating the vascular system for the purpose of operational maintenance of stimulated neurons (Baslow and Guilfoyle, 2007), has led to new insights into the possible nature of the modular repeating structural and functional units of the brain. These insights have resulted in the formation of two hypotheses. First, that the repeating "structural unit" in the brain is defined by the tri-cellular metabolism of NAA and NAAG, operating within a four-cell physiological domain that includes vascular endothelial cells, and consists of a single neuron and one or more oligodendrocytes, astrocytes, and endothelial cells. Second, that based on the two-cell biosemiotic model which proposes that only two neurons are required for information transmission, learning, and the formation of memory engrams, that a "functional unit" in brain consists of two structural units (Baslow, 2010a). In the following sections, a rationale for these hypotheses is provided.

\section{DISCUSSION}

\section{EVIDENCE OF THE BRAIN'S PHYSIOLOGICAL OPERATING SYSTEM The tri-cellular metabolism of NAA and NAAG}

$N$-acetylaspartate and NAAG are substances synthesized by, and present in great abundance in neurons, whose possible functions have been the subject of research efforts over a period of many decades (Baslow and Guilfoyle, 2006). NAA is an N-acetylated derivative of L-aspartic acid (Asp), and NAAG is a dipeptide derivative of NAA, in which Glu is joined to the Asp moiety via a peptide bond. Glucose (Glc) is the on-going source of the acetyl-coenzyme A (AcCoA) used for the acetylation of Asp, and NAA is the only precursor of NAAG. Thus, NAA and NAAG are metabolically linked substances that are synthesized by neurons as a function of their rate of Glc utilization. However, neurons cannot catabolize either of these substances, so that their metabolism requires the participation of three cell types for its completion, forming a tri-cellular metabolic unit (Baslow, 2000). These cells include neurons, where NAA and NAAG are synthesized and then released to ECF upon stimulation; astrocytes, target cells expressing the metabotropic Glu receptor-3 (GRM3) for NAAG, and which can hydrolyze NAAG but not NAA, and oligodendrocytes; the target cells for NAA, which can hydrolyze NAA but not NAAG. In this metabolic cycling, the NAA by-product of astrocyte NAAG hydrolysis, along with NAA exported directly to
ECF by neurons, are both hydrolyzed by oligodendrocytes liberating Asp that is recycled to neurons, and acetate (Ac), that is further metabolized by oligodendrocytes. The hydrolyzed Glu is converted into glutamine (Gln) by astrocytes and recycled to neurons. This sequence is unique in that it is the only tri-cellular amino acid metabolic cycle known to occur in brain, and with neurons, astrocytes and oligodendrocytes comprising approximately $75 \%$ of the volume of the brain and more than $95 \%$ of its cellular volume (Baslow, 2007), the tri-cellular metabolic cycling of NAA and NAAG is an important global feature of metabolism in brain. Paradoxically, this cycling is apparently unnecessary for either neuron survival or for generation of action potentials in isolated neurons, as evidenced by loss of NAA in cultivated brain slices (Baslow et al., 2003), nor in the intact brain as evidenced by the singular human case where NAA and NAAG are both absent (Martin et al., 2001).

\section{Released NAA and NAAG are involved in the export of metabolic water}

Both NAA and NAAG released to ECF down their gradients upon neurostimulation are associated with a large number of obligated water molecules which effectively results in the transport of these osmolyte-bound water molecules to ECF against a water gradient (Baslow, 1999a). However, these bound water molecules cannot be easily removed from ECF unless they are first released as free water. Thus, in addition to catabolizing NAA by oligodendrocyte aspartoacylase (ASPA) as part of the tri-cellular NAA-NAAG metabolic cycle, this enzyme also serves as the release mechanism for NAA-bound water which is then liberated to ECF as free water upon hydrolysis of NAA and the cellular uptake of its Ac and Asp moieties. The liberated free water can then be removed from the brain down a water gradient via aquaporin 4 (AQP4) "free" water channels present on the surfaces of astrocytes and vascular endothelial cells followed by vascular system transport of the water out of the brain. Neurons require such an osmolyte driven molecular water pump (MWP) mechanism (Baslow, 2002) since they are insulated from the movement of free water in that they do not express AQP4 channels (Agre et al., 2002), and do not form gap junctions ( $G j$ ) with any of their chaperone glial cells for the movement of either osmolyte-bound or free water (Rash et al., 1997). The minimum number of obligated water molecules associated with each exported NAA and NAAG molecule as "bound" water is 32 and 53 molecules respectively. The tri-cellular metabolism of NAA and NAAG and its association with the transport of water is shown in Figure 1.

\section{A four-cell physiological unit, the brain's "operating system"}

The unique tri-cellular metabolism of NAA and NAAG serves an important physiological function in brain, and that is to couple the activity of neurons with their metabolic needs. To do this, the tricellular metabolism of NAA and NAAG operates in a four-cell physiological domain. Upon stimulation, NAA and NAAG are released to ECF. The NAA is hydrolyzed by oligodendrocytes, liberating free water for removal from the brain. NAAG that is released first docks with its target, the astrocyte GRM3 receptor, where the GRM3-associated NAAG peptidase then releases the Glu, activating the receptor and initiating $\mathrm{Ca}^{++}$waves in astrocytes (Baslow, 2010b). The physiological results of NAA and NAAG release down their gradients and their recycling are twofold. First, stimulation-released NAA and NAAG with their obligated water both play a role in maintaining neuronal 
osmotic and structural integrity by transporting metabolic and other water to ECF against a water gradient for its removal from the brain (Baslow, 2007). Second, the hydrolysis of NAAG and liberation of Glu initiates astrocyte-mediated signaling via release of second messengers to capillary endothelial cells which in turn induce a focal hyperemic response. This coupling of the rate of neuronal activity directly with the rate of blood flow serves to provide stimulated neurons with the energy required to transmit meaningful frequencyencoded messages as well as with increased sink capacity for metabolic water and $\mathrm{CO}_{2}$ (Baslow and Guilfoyle, 2007). As the outcome of this homeostatic process provides neurons with required energy and the timely removal of waste products, this four-cell physiological unit has been proposed to constitute the "operating system" in brain, and is considered to be essential for maintenance of normal cognitive and motor functions (Baslow, 2010c).

\section{Inborn errors in the metabolism of NAA and NAAG affect the brain's "operating system"}

Evidence based on the outcomes of two human inborn errors in NAA and NAAG metabolism strongly support the hypothesis that NAA and NAAG metabolism and their physiological roles constitute the brain's operating system (Baslow and Guilfoyle, 2007). The etiology of Canavan disease (CD or hyperacetylaspartia) has been traced to mutations that inactivate ASPA (Matalon, et al., 1988), the enzyme that catabolizes NAA and also serves as its water release mechanism (Baslow, 1999b). The lack of ASPA activity appears to be responsible for $\mathrm{CD}$, and in this early-onset syndrome, there is a global buildup of NAA, NAAG, and water in brain that is associated with severe osmotic consequences including megalocephaly and spongiform degeneration in white matter as well as with profound cognitive and motor failures. The second inborn error in NAA and NAAG metabolism is a singular known case where there is a lack of NAA and NAAG in brain (hypoacetylaspartia; Martin et al., 2001; Boltshauser et al., 2004). In this case, the neuronal NAA synthase that joins AcCoA with Asp is inactive (Wiame et al., 2010), resulting in the inability of neurons to synthesize NAA, and therefore in the absence of both NAA and its adduct NAAG in brain. In this singular case, the entire NAA-NAAG metabolic and physiological operating system is inactivated, essentially decoupling neuronal activity from its interaction with the vascular system. The results of this early-onset genetic disease are also profound as evidenced by both cognitive impairment and severe motor disturbances in this individual. The outcomes of these two inborn errors in metabolism of

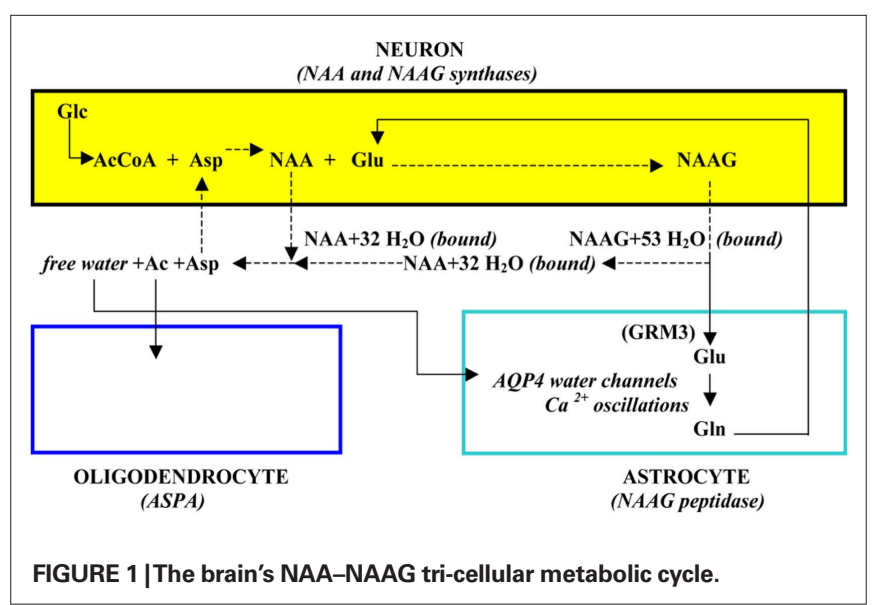

NAA support the notion that the tri-cellular metabolism of NAA and NAAG, and their physiological interactions with the vascular system, play a vital role in the normal operation of the brain.

\section{THE STRUCTURAL UNIT OF THE BRAIN}

Incorporating what is known about the tri-cellular metabolism of NAA and NAAG, along with its role in the brain's physiological operation that couples neuronal activity with the energy required to maintain the ability of neurons to function at all levels of stimulation, it is possible to reflect on the nature of the basic structural unit that is present in brain. Based on these considerations, it has been proposed that the NAA-NAAG tri-cellular metabolic system, operating in a four-cell physiological domain that includes vascular epithelial cells, defines the minimal unit of brain structure. This structural unit is illustrated in Figure 2.

In this cartoon, the three cell types involved in the NAA-NAAG metabolic cycle as well as the four-cell physiological domain in which it operates are shown. Since NAA and NAAG synthesis and their stimulation-induced release is an inherent property of every neuron, a "structural unit" of the brain is considered to be formed from a single neuron and one or more astrocytes, oligodendrocytes and endothelial cells, and it is proposed that it is the repetition of these structural modules that form the vertebrate "brain".

\section{THE FUNCTIONAL UNIT OF THE BRAIN}

\section{The two-cell biosemiotic model and evidence of neuronal words and language}

The method used by a single neuron to transmit frequency-encoded information is by generation of wave-like spikes (S) or depolarization's and rapid re-polarization's of its plasma membrane with an average depolarization/re-polarization time of about $1 \mathrm{~ms}$. The spike re-polarization time also constitutes the absolute refractory

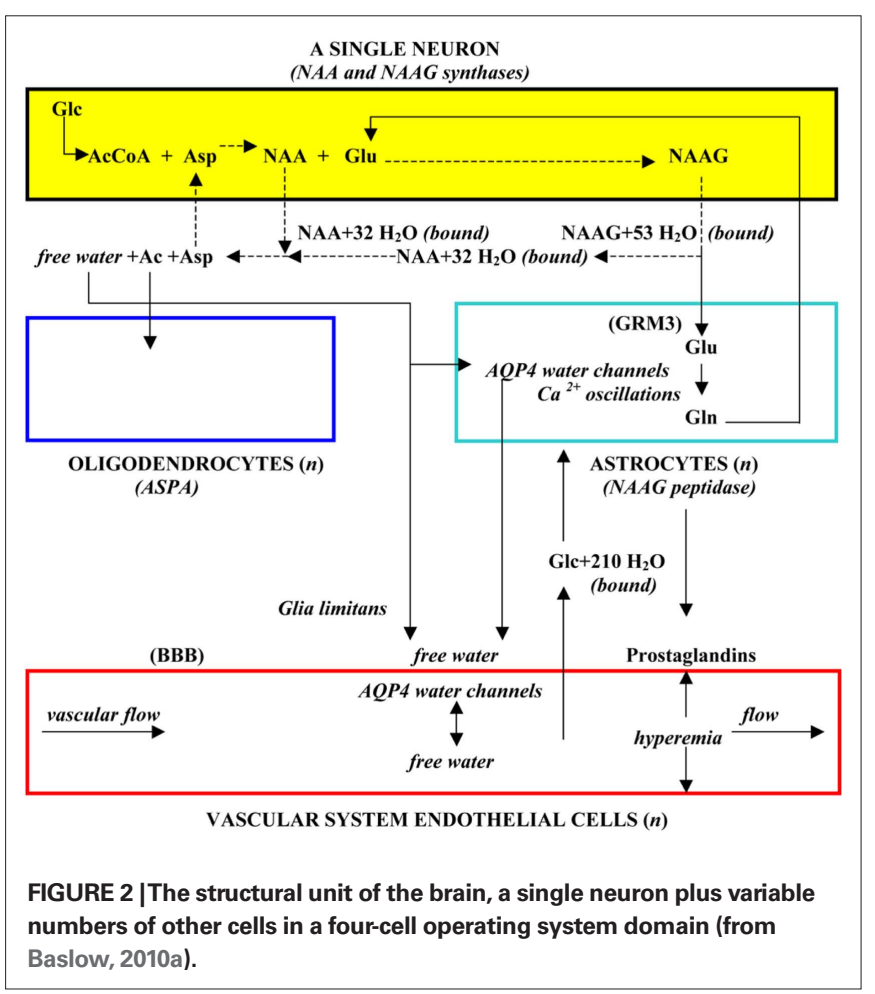


period during which time another spike cannot be generated. This is followed by a relative refractory period of $1-2 \mathrm{~ms}$ where a strong signal can stimulate another spike. Spikes are also interspersed with longer pauses generally lasting from 0 to $10000 \mathrm{~ms}$. Thus, a spike of $1 \mathrm{~ms}$ followed by a total pause (P) of $9 \mathrm{~ms}$ lasts $10 \mathrm{~ms}$ and its repetition would represent neuronal signaling at a frequency of $100 \mathrm{~Hz}$. Although frequency and S/P words are related, each spike in an S/P word is physiologically meaningful in that it has a time dimension, absent from frequency notations, that allows for interactions with mechanisms for release of quanta of neurotransmitters at synapses

Table 1 | List of some neuronal S/P words and their meanings (adapted from Baslow, 2010a).

\begin{tabular}{|c|c|c|c|c|c|}
\hline Organism & Sensor & Word & & Meaning & Reference \\
\hline \multicolumn{6}{|l|}{ BLOWFLY } \\
\hline \multirow[t]{5}{*}{ Sensillum } & Salt & $\mathrm{S}_{1} \mathrm{P}_{499}$ & $\mathrm{NaCl}(\mathrm{M})$ & 0.10 & Gillary (1966) \\
\hline & & $\mathrm{S}_{1} \mathrm{P}_{45}$ & & 0.20 & \\
\hline & & $\mathrm{S}_{1} \mathrm{P}_{27}$ & & 0.25 & \\
\hline & & $\mathrm{S}_{1} \mathrm{P}_{18}$ & & 0.50 & \\
\hline & & $\mathrm{S}_{1} \mathrm{P}_{7}$ & & 3.00 & \\
\hline \multicolumn{6}{|c|}{ GROUND BEETLE } \\
\hline \multirow[t]{3}{*}{ Sensillum } & Salt & $S_{1} P_{1000}$ & $\mathrm{NaCl}(\mathrm{M})$ & 0.001 & Merivee et al. (2004) \\
\hline & & $S_{1} P_{71}$ & & 0.01 & \\
\hline & & $\mathrm{S}_{1} \mathrm{P}_{29}$ & & 0.10 & \\
\hline \multirow{3}{*}{ Taste cells } & & $\mathrm{S}_{1} \mathrm{P}_{312}$ & & 3.0 & \\
\hline & & $\mathrm{S}_{1} \mathrm{P}_{1999}$ & & 4.3 & \\
\hline & & $S_{1} P_{4000}$ & & 5.0 & \\
\hline
\end{tabular}

\section{CAT}

Hypothalamus

glucosensing

\section{Glucose}

Steady-state

Glc i.v. 30 min

\section{RAT}

Taste cells

RAT

Hippocampal neuron

Quinine

Sound

GUINEA PIG

GABAergic neurons

HONEYBEE

Vibration-sensing

interneuron

\section{GRASSHOPPER}

Auditory receptor cells

RAT

Head direction cells

$$
S_{1} P_{4000}
$$

\subsection{1}

0.10

1.00

\begin{abstract}
(Syn). Information is transmitted in the form of these $\mathrm{S} / \mathrm{P}$ sequences and it has been proposed that these transmissions constitute natural neuronal languages in an S/P "word” form (Baslow, 2009, 2010d). The only reason for an individual neuron to generate an $\mathrm{S} / \mathrm{P}$ word would be to transmit information to another neuron that can receive, interpret, and respond to the message. Therefore, the minimal cellular unit for such transmissions must consist of two neurons. Using an average spike time of $1 \mathrm{~ms}$ applied to frequency-based data, some examples of neuronal S/P words generated by individual neurons, and their meanings in different organisms are presented in Table 1.
\end{abstract} (1) 
Since the spike-generation process is metabolically costly, it requires that energy be constantly replenished or the timing of the spike-pause periods will be altered, and meaningful frequency-encoded information lost. From this table, it is apparent that a delay in "P" of only a few ms can significantly alter the meaning of a $\mathrm{S} / \mathrm{P}$ word.

\section{A functional unit of the brain consists of two structural units}

Whereas the structural unit presented in Figure $\mathbf{1}$ is considered to be the basic unit of brain formation, the basic unit of brain function requires two neurons as proposed in the "two-cell" model of neuron communication (Baslow, 2009). Based on that model, two neurons are not only required as the minimal unit for cell to cell communication, a sending neuron (afferent) and a receiving neuron (efferent) joined by their complex and malleable interconnecting DSD fields, but are also deemed to be sufficient for all aspects of information processing. The two-cell model of neuron communication as described contains all of the dendritic, synaptic, and enzymatic elements found throughout the brain that are required for transmission of meaningful frequency-encoded information, as well as for learning, and formation of long-term memory engrams. This model is therefore proposed to be the basic unit of cognition in brain. Since two neurons are required for function, it follows that cognitive ability is an inherent property of each binary set of structural units. Between structural units, neurons are connected to each other at synapses (Syn), and astrocytes are connected to one another, and to oligodendrocytes via gap junctions $(G j)$. The endothelial cells are connected to each other by tight junctions ( $T j$ ) forming the capillary channels of the vascular system, and lastly, the ECF between cells forms contiguous channels, constituting a common highway for neurotransmitters, gliotransmitters, and traffic of other imported and exported substances. In Figure 3, two structural units of brain representing one functional unit is illustrated.

\section{THE MACROSTRUCTURE OF THE BRAIN IS A FUNCTION OF ITS STRUCTURAL AND FUNCTIONAL UNITS}

Based on the proposed structural and functional units in brain, the macrostructure of the brain can be envisioned to be composed of the three-dimensional stacking and interlinking of individual structural units arranged in multiple binary functional units. However, because of the property of neurons to form elongated axonal extensions and dendritic arbors that can interact with multiple neurons, structural units that are interconnected in binary pairs may exhibit many configurations and can be long distances from one another in three-dimensional brain space. As an added complexity, neurons in these binary structural units can use one or more very different neurotransmitters at their synaptic connections, some excitatory and some inhibitory. Nonetheless, since the NAA and NAAG metabolic system is found in all types of neurons, it is the operation of this tri-cellular metabolic system repeated in every structural unit, and its four-cell physiological role that defines the nature of a binary functional unit rather than neuronal morphology or the neurotransmitter types used between its component structural units. In Figure 4, nine associated structural units are shown, each containing its own tri-cellular metabolic unit, each operating in a four-cell physiological domain and emphasizing the metabolic, physiological, and operational unity of each structural module. The possible combinations and permutations of functional units even in this small sample could be very large, and each binary functional unit would be capable of meaningful communication, as well as "learning" and "memory engram formation" and thus be able to participate in the overall information processing activities of the brain. In brain, these units are intertwined, stretched, and twisted to form the many different brain regions, and including both gray and white matter, that comprise the whole organ.

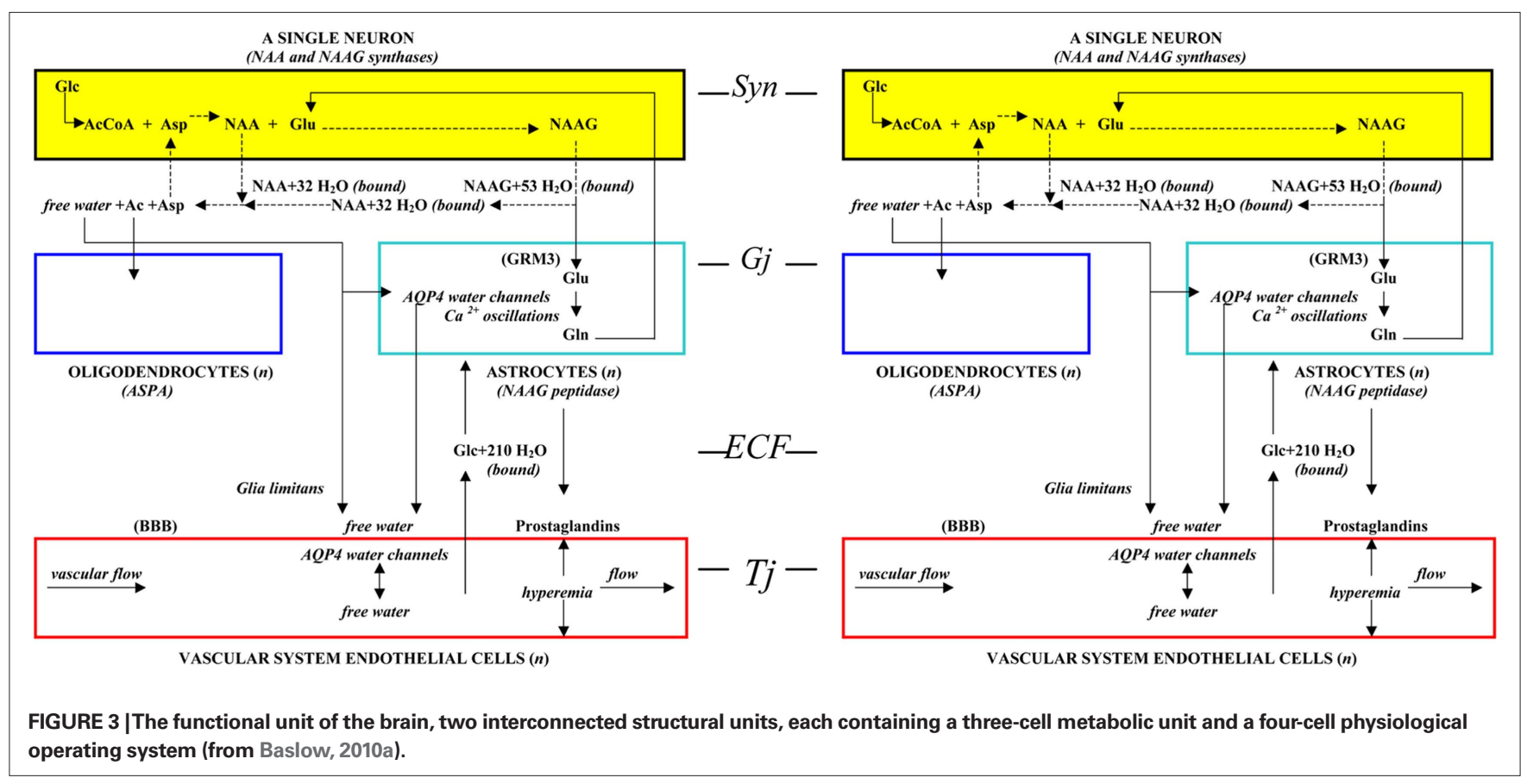




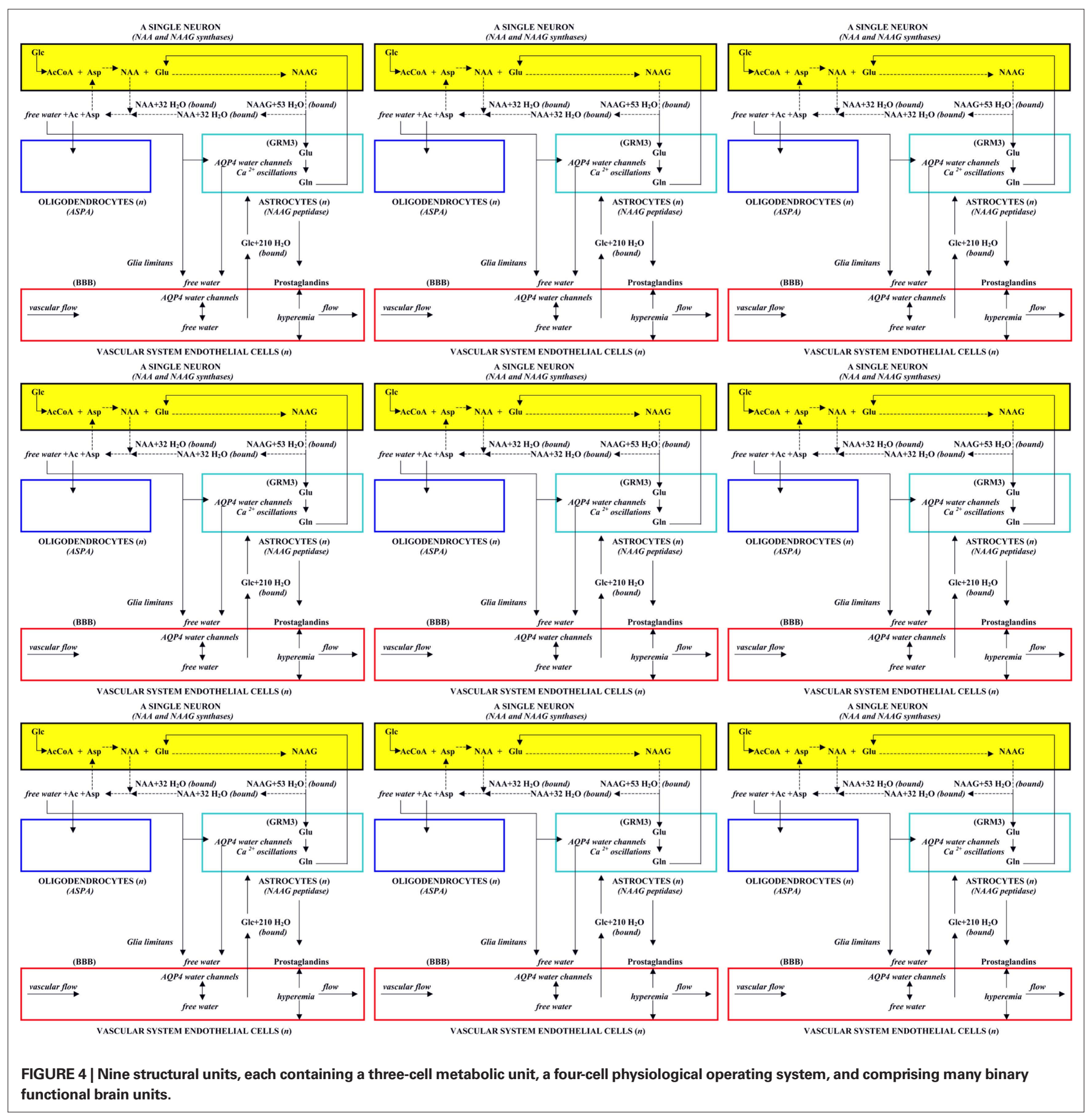

\section{CONCLUSION}

In this article an attempt has been made to understand the nature of the brain's hierarchical modular repeating cellular units for both structure and function based on the identification of two fundamental processes in brain. One is based on evidence of the brain's operating system, a metabolic-physiological process that couples neuronal activity directly with focal blood flow, and the other is based on evidence that individual neurons use timing-dependent specific words and language to communicate. The elucidation of these global brain processes has provided evidence-based insights and a rationale for describing the basic nature of the modular repeating structural and functional units of the brain. These insights have led to two conclusions. First, that the repeating "structural unit" in the brain is defined by the tri-cellular metabolism of NAA and NAAG operating within a four-cell physiological domain that includes one neuron, and at least one each of oligodendrocytes, astrocytes, and vascular endothelial cells. Second, that based on the two-cell biosemiotic model, that the basic "functional unit" in brain consists of two neurons and their interconnecting DSD field. Since a functional unit consists of two neurons, two structural 
units are required to form a functional unit. Thus, the brain can be envisioned as being made up of the three-dimensional stacking and intertwining of myriad structural units which results not only in its gross structure, but also in producing a uniform distribution of functional units. Since the NAA-NAAG physiological operating system that couples neuronal activity with focal blood flow is repeated in every structural unit, it is global in nature and therefore well positioned to control normal brain activities that underlay complex outcomes such as those associated with establishment

\section{REFERENCES}

Agre, P., King, L. S., Yasui, M., Guggino, W. B., Ottersen, O. P., Fujiyoshi, Y., Engel, A., and Neilsen, S. (2002). Aquaporin water channels-from atomic structure to clinical medicine. J. Physiol. 542, 3-16.

Ai, H., Rybak, J., Menzel, R., and Itoh, T. (2009). Response characteristics of vibration-sensitive interneurons related to Johnston's organ in the honeybee, Apis mellifera. J. Comp. Neurol. 515, 145-160.

Anand, B. K., Chhina, G.S., Sharma, K. N., Dua, S., and Singh, B. (1964). Activity of single neurons in the hypothalamic feeding centers: effect of glucose. Am. J. Physiol. 207, 1146-1154.

Barbieri, M. (2009). A short history of biosemiotics. Biosemiotics 2, 221-245.

Baslow, M. H. (1999a). The existence of molecular water pumps in the nervous system. A review of the evidence. Neurochem. Int. 34, 77-90.

Baslow, M. H. (1999b). Molecular water pumps and the etiology of Canavan disease; a case of the sorcerer's apprentice. J. Inherit. Metab. Dis. 22, 99-101.

Baslow, M. H. (2000). Functions of $\mathrm{N}$-acetyl-L-aspartate and N-acetyl$\mathrm{L}$-aspartylglutamate in the vertebrate brain. Role in glial cell-specific signaling. J. Neurochem. 75, 453-459.

Baslow, M. H. (2002). Evidence supporting a role for $\mathrm{N}$-acetyl-L-aspartate as a molecular water pump in myelinated neurons in the central nervous system. An analytical review. Neurochem. Int. 40, 295-300.

Baslow, M. H. (2007). "N-acetylaspartate and N-acetylaspartylglutamate," in Handbook of Neurochemistry and Molecular Neurobiology, 3rd Edn, Vol. 6, Amino Acids and Peptides in the Nervous System, ed. A. Lajtha (New York, NY: Springer Science), 305-346.

Baslow, M. H. (2009). The languages of neurons: an analysis of coding mechanisms by which neurons communicate, learn and store information. Entropy 11, 782-797.
Baslow, M. H. (2010a). Biosemiosis and the cellular basis of mind. How the oxidation of glucose by individual neurons in brain results in meaningful communications and in the emergence of "mind". Biosemiotics, 4, 1-15.

Baslow, M. H. (2010b). A novel key-lock mechanism for inactivating amino acid neurotransmitters during transit across extracellular space. Amino Acids 38, 51-55.

Baslow, M. H. (2010c). Evidence that the tri-cellular metabolism of $\mathrm{N}$-acetylaspartate functions as the brain's “operating system": how NAA metabolism supports meaningful intercellular frequency-encoded communications. Amino Acids 39, 1139-1145.

Baslow, M. H. (2010d). The nature of neuronal words and language. Nat. Sci. 2, 205-211.

Baslow, M.H., and Guilfoyle, D. N. (2006). Functions of $\mathrm{N}$-acetylaspartate and $\mathrm{N}$-acetylaspartylglutamate in brain: evidence of a role in maintenance of higher brain integrative activities of information processing and cognition. Adv. Exp. Med. Biol. 576, 95-112.

Baslow, M.H., and Guilfoyle, D. N. (2007). Using proton magnetic resonance imaging and spectroscopy to understand brain "activation". Brain Lang. 102, 153-164.

Baslow, M. H., Suckow, R. F., Gaynor, K., Bhakoo, K. K., Marks, N., Saito, M., Saito, M., Duff, K., Matsuoka, Y., and Berg, M. (2003). Brain damage results in downregulation of $\mathrm{N}$-acetylaspartate as a neuronal osmolyte. Neuromolecular Med. 3, 95-103.

Boltshauser, E., Schmitt, B., Wevers, R. A., Engelke, U., Burlina, A. B., and Burlina, A. P. (2004). Follow-up of a child with hypoacetylaspartia. Neuropediatrics 35, 255-258.

Di Lorenzo, P. M., Leshchinskiy, S., Moroney, D. N., and Ozdoba, J. M. (2009). Making time count: functional evidence for temporal coding of taste sensation. Behav. Neurosci. 123, 14-25.

of an individual's "mind", the momentary aggregate of all natural brain processes involving perception, thought, insight, foresight, and imagination (Barbieri, 2009), and of specific "behaviors", outward manifestations of the moment to moment trajectory of a mind. As a corollary, any malfunction in any of the metabolicphysiological components of the brain's operating system, as are observed in $\mathrm{CD}$ and hypoacetylaspartia, or in any of the signaling components of binary functional units, would be expected to result in some degree of abnormal brain functioning.

Djurfeldt, M., Ekeberg, O., and Lansner, A (2008). Large-scale modeling - a tool for conquering the complexity of the brain. Front. Neuroinform. 2:1. doi: 10.3389/neuro.11.001.2008

Eyherabide, H. G. ., Rokem, A., Herz, A. V. M., and Samengo, I. (2009). Bursts generate a non-reducible spike-pattern code. Front. Neurosci. 3:1. doi: 10.3389/ neuro.01002.2009

Gilbertson, T. A., Avenet, P., Kinnamon, S. C., and Roper, S. D. (1992). Proton currents through amiloride-sensitive $\mathrm{Na}$ channels in hamster taste cells. $J$. Gen. Physiol. 100, 803-824.

Gillary, H. L. (1966). Stimulation of the salt receptor of the blowfly. II. Temperature. J. Gen. Physiol. 50, 351-357.

Lee, C. R., and Rice, M. E. (2008). Hydrogen peroxide increases the excitability of substantia nigra pars reticulata GABAergic neurons. Society for Neuroscience Meeting, November 16, 2008, Program 179.2, Poster No. QQ37.

Martin, E., Capone, A., Schneider, J., Hennig, J., and Thiel, T. (2001). Absence of $\mathrm{N}$-acetylaspartate in the human brain: impact on neurospectroscopy? Ann. Neurol. 49, 518-521.

Matalon, R., Michals, K., Sebasta, D., Deanching, M., Gashkoff, P., and Casanova, J. (1988). Aspartoacylase deficiency and $\mathrm{N}$-acetylaspartic aciduria in patients with Canavan disease. Am. J. Med. Genet. 29, 463-471.

Merivee, E., Renou, M., Mand, M.,Luik,A., Heidemaa, M., and Ploomi, A. (2004). Electrophysiological responses to salts from antennal chaetoid taste sensilla of the ground beetle Pterostichus aethiops. J. Insect Physiol. 50, 1001-1013.

Meunier, D., Lambiotte, R., Fornito, A., Ersche, K. D., and Bullmore, E. T. (2009). Hierarchical modularity in human brain functional networks. Front. Neuroinform.3:37. doi: 10.3389/ neuro.11.037.2009

Rash, J. E., Duffy, H. S., Dudek, F. E., Bilhartz, B. L., Whalen,, L. R., and Yasumura, T. (1997). Grid-mapped freeze-fracture analysis of gap junctions in gray and white matter of adult rat central nervous system, with evidence for a "panglial syncytium" that is not coupled to neurons. J. Comp. Neurol. 388, 265-292.

Takahashi, S., and Sakurai, Y. (2009). Sub-millisecond firing synchrony of closely neighboring pyramidal neurons in hippocampal CA1 of rats during delayed non-matching to sample task. Front. Neural Circuits 3:9. doi: 10.3389/neuro.04.009.2009

Taube, J. S. (2010). Interspike interval analyses reveal irregular firing patterns at short, but not long, intervals in rat head direction cells. J. Neurophysiol. 104, 1635-1648.

Wiame, E., Tyteca, D., Pierrot, N., Collard, F., Amyere, M., Noel, G., Desmedt, J., Nassogne, M-C., Vikkula, M.,Octave, J-N., Vincent, M-F., Courtoy, P. J., Boltshauser, E., and Van Schaftingen, E. (2010). Molecular identification of aspartate $\mathrm{N}$-acetyltransferase and its mutation in hypoacetylaspartia. Biochem. J. 425, 127-136.

Conflict of Interest Statement: The author declares that preparation of this manuscript was conducted without any commercial or financial interests that could be interpreted as a conflict of interest.

Received: 10 December 2010; accepted: 25 January 2011; published online: 15 June 2011.

Citation: Baslow MH(2011) The vertebrate brain, evidence of its modular organization and operating system: insights into the brain's basic units of structure, function, and operation and how they influence neuronal signaling and behavior. Front. Behav. Neurosci. 5:5. doi: 10.3389/ fnbeh.2011.00005

Copyright (C) 2011 Baslow. This is an openaccess article subject to a non-exclusive license between the authors and Frontiers Media SA, which permits use, distribution and reproduction in other forums, provided the original authors and source are credited and other Frontiers conditions are complied with. 